\title{
Single particle ICP-MS for the analysis of nanoparticles and colloids in the environment
}

\author{
MUAMMAR MANSOR ${ }^{1}$, ANKITA CHAUHAN ${ }^{1}$, SÖREN \\ DRABESCH $^{1}$, ANH VAN LE $^{2}$, TIMM BAYER ${ }^{3}$ AND \\ ANDREAS KAPPLER ${ }^{4}$ \\ ${ }^{1}$ University of Tuebingen \\ ${ }^{2}$ Geomicrobiology Group - Center for Applied Geoscience \\ (ZAG) - Eberhard-Karls-University Tuebingen \\ ${ }^{3}$ University of Tuebingen, Center for Applied Geoscience \\ ${ }^{4}$ Eberhard-Karls-University Tuebingen \\ Presenting Author: muammar.muammar-bin-mansor@uni- \\ tuebingen.de
}

Aquatic natural nanoparticles $(\leq 100 \mathrm{~nm})$ and colloids $(\leq 1,000$ $\mathrm{nm}$ ) constitute a highly dynamic environmental pool of particles with a wide continuum in size, reactivity and transport properties. At the same time, engineered and incidental nanoparticles and colloids originating from anthropogenic activities are being released at an expanding rate into the environment. Our knowledge of the particles' dynamic influence on biogeochemical cycling is limited by the techniques available at our disposal. Here we introduce single particle ICP-MS (spICP-MS), which is an emerging tool that provides the elemental composition of a single particle upon ionization in the plasma. Sample preparation is easy - often requiring only dilutions - and thousands of particles can be analyzed within minutes. By providing information at both the single-particle (particle mass and size distribution) and the population level (particle number and mass concentrations, average particle mass and size), spICP-MS neatly fills the informational gap between widely used techniques such as electron microscopy and sequential filtration. In this work, we present three examples for the application of spICP-MS, from the simple case of synthetic nanoparticle (abiogenic and biogenic magnetite) aggregation, to colloidal contaminant (arsenic) transport, and finally to the elemental association (iron-organic matter associates) within complex natural samples. We end by discussing strategies for sample manipulation and data interpretation, highlighting the unique insights and challenges when applying spICP-MS to the analysis of nanoparticles and colloids in environmental systems.

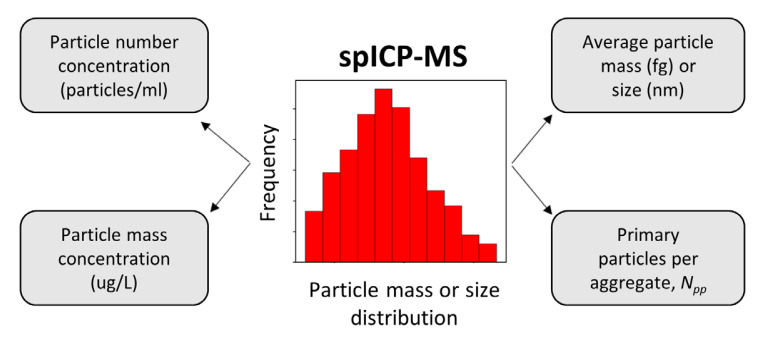

